\title{
Inteligencia para la Seguridad Pública en las Entidades Federativas de México
}

\author{
Intelligence for Public Security in Mexican States \\ JUAN CARLOS MONTERO BAGATELLA \\ Escuela de Ciencias Sociales y Gobierno del Tecnológico de Monterrey, Campus \\ Monterrey, México
}

\begin{abstract}
RESUMEN: La seguridad pública en México continúa siendo uno de los problemas más importantes. La inseguridad, expresada en estadísticas sobre incidencia delictiva y encuestas sobre percepción de inseguridad, no se ha logrado reducir. Ante dicho escenario, el artículo expone la complejidad de la seguridad pública, reconociéndola como una materia que trasciende las atribuciones de distintas dependencias y órdenes de gobierno. La investigación se enfoca en el papel de la inteligencia estratégica en las entidades federativas, reconociéndola como instrumento para la toma de decisiones. Para ello, el trabajo comienza por exponer la complejidad de la seguridad pública de acuerdo con el marco legal; en segundo lugar, se expone la utilidad de la inteligencia y de la inteligencia estratégica para la seguridad pública; en tercer lugar, se expone la investigación realizada en las 32 entidades federativas para identificar sus capacidades para generar y compartir inteligencia para la seguridad pública. La investigación expone la falta de mecanismos para generar y compartir inteligencia al interior de las entidades federativas y entre órdenes de gobierno, así como una visión restringida de la inteligencia orientada hacia la función policial, excluyendo elementos como la prevención de la violencia y el delito o la reinserción social.
\end{abstract}

PALABRAS ClaVE: Inteligencia estratégica, Seguridad pública, Función policial, Entidades federativas, Seguridad en México.

ABSTRACT: Security in Mexico is still being one of the most problematic issues to address. Incidence of crime statistics and perception of insecurity surveys show that insecurity has not been reduced. In this context, the article shows the complexity of public security as an issue that involves the work of different governments but also different institutions withing governments. The research focused on the role of strategic intelligence as an instrument for decision making in public security issues in Mexican states. In this way, the article begins by showing the complexity of the public security issues according with the legal frame. In second place, strategic intelligence is presented as an instrument to address public security. In third place, the article shows the results of the research conducted to identify the capabilities to produce and share intelligence in all Mexican states. The main contribution is to identify the lack of mechanisms to produce and share intelligence in Mexican states but also the limited vision of intelligence as an issue related only to policing instead of considering other elements of public security such as prevention of violence and crime or social reintegration of criminals.

KEYwords: Strategic intelligence, Public security, Policing in Mexico, Mexican states, Security in Mexico.

Recibido: 1 de julio de 2020. Aceptado: 21 de octubre de 2020.

Revista de Estudios en Seguridad Internacional, Vol. 6, No. 2, (2020), pp. 193-213. http://www.seguridadinternacional.es/revista/

ISSN: 2444-6157. DOI: http://dx.doi.org/10.18847/1.12.11 


\section{INTRODUCCIÓN}

La inseguridad y la violencia se han convertido en algunos de los problemas más importantes en México. La gestión del gobierno federal de la administración 2018-2024 ha enfrentado el mayor número de homicidios en México en años recientes y la estrategia gubernamental no ha impactado en la estadística delictiva, ni en la percepción de violencia. Si bien, a partir del mes de septiembre del 2019 se comenzó a observar un descenso en el número de homicidios y la percepción de inseguridad en México, de acuerdo con cifras provistas por el Secretariado Ejecutivo del Sistema Nacional de Seguridad Pública (SESNSP) (SESNSP, 2020), dicho descenso ha sido marginal y no muestra que la inseguridad y la percepción de violencia estén sufriendo una tendencia negativa (INEGI, 2020). En cambio, las masacres de policías como la ocurrida en el mes de octubre en Michoacán; o en noviembre en el estado de Oaxaca; así como el enfrentamiento armado que se vivió en la ciudad de Culiacán, Sinaloa, el jueves 18 de octubre, la masacre a la familia LeBarón en el estado de Sonora, todos ellos durante el 2019, la violencia en el estado de Guanajuato y el intento de asesinato al Secretario de Seguridad de la Ciudad de México, durante el 2020, exponen las limitaciones gubernamentales para enfrentar el problema y también exponen un problema nacional (crimen organizado) con expresiones subnacionales.

La seguridad es un tema complejo, cuya definición conceptual, enfoque y atribuciones legales están determinados por la adjetivación del concepto. La variedad de enfoques con que se analiza y la estructura jurisdiccional define temas transversales y complejos. En consecuencia, la seguridad constituye una materia que requiere una gobernanza multinivel, con la participación de distintos actores gubernamentales y sociales, apoyados en la coordinación de diferentes órdenes de gobierno (Clarke \& Chenoweth, 2006; GonzálezLópez, 2019).

La complejidad de la gobernanza de la seguridad pública en México depende de diferentes variables y recursos, entre los que se encuentran la división política entre los órdenes de gobierno, la disponibilidad de recursos financieros, pero también los modelos de gestión administrativa. Respecto a este último punto, un modelo innovador que promueve la eficiente gestión de los recursos para la seguridad pública, especialmente en contextos de limitación presupuestal o en el estado de fuerza policial (Darroch \& Mazerolle, 2013), es el de la acción policial guiada por inteligencia (Intelligence-led Policing) o ILP por sus siglas en inglés. Este modelo surgió en la década de nos 90 en el Reino Unido, pero sería hasta después de los atentados terroristas del 11 de septiembre del 2001 en los Estados Unidos que se difundiría ampliamente en distintos países al reconocer la importancia de la generación de inteligencia por parte de los gobiernos subnacionales y su colaboración con los gobiernos nacionales en el combate al terrorismo (Gemke et al., 2019), pero que se han convertido en una herramienta de gestión para la seguridad, reconociendo la complejidad de la criminalidad (Carter, 2016; Johnson, 2010).

La información dispersa o sin analizar limita a los tomadores de decisiones de ejercer su función (Gookins, 2003). Para lograr la eficiencia en la toma de decisiones y en la planeación estratégica de la actuación de la autoridad, resulta necesario el desarrollo de áreas, procesos y modelos de gestión cuyos productos estén orientados al fortalecimiento de las líneas de acción para la prevención y reducción del delito, así como a la generación de insumos para fortalecer la actuación policial, lo cual se expresa en el diseño de políticas de seguridad pública basadas en evidencia. Frente a este reto, la ILP se constituye como un modelo de 
gestión con herramientas basadas en evidencia para alcanzar los objetivos de la seguridad, apoyando los procesos de toma de decisiones para la reducción y prevención del crimen (Bureau of Justice Assistance, 2010; Carter, 2016; Darroch \& Mazerolle, 2013). Si bien la ILP puede entenderse como un modelo restringido a la función policial, en realidad constituye un modelo de gestión de la seguridad, que tiene por objetivo no la reacción sino la prevención de la violencia y el delito de una manera proactiva, por lo que se apoya de manera importante en el reconocimiento de la necesidad de que dependencias no relacionadas directamente con el combate al crimen, participen en la prevención.

La presente investigación parte del reconocimiento de que los actos criminales son conducidos por actores cuyas conductas no se circunscriben a los límites jurisdiccionales (Treverton, 2011); en cambio, la seguridad pública es una materia multidimensional que requiere de una gobernanza multinivel (González-López, 2019), lo que implica la necesidad de coordinación a niveles intragubernamental e intergubernamental. Al respecto, la definición de seguridad pública en México se encuentra en el noveno párrafo del artículo $21^{\circ}$ de la Constitución Política de los Estados Unidos Mexicanos, en donde señala que "La seguridad pública comprende la prevención, investigación y persecución de los delitos, así como la sanción de las infracciones administrativas...", señalando también que es una función del Estado a cargo de la federación, las entidades federativas y los municipios; en consecuencia, la prevención, investigación, persecución y sanción de los delitos se encuentra dividido jurisdiccionalmente entre los distintos órdenes de gobierno, así como entre distintas dependencias de cada orden de gobierno, lo que expone la definición de la seguridad pública como una materia que implica una gobernanza multinivel y la coordinación intragubernamental e intergubernamental. Entre los actores que participan en la política de seguridad pública en México se encuentran los gobiernos subnacionales, destacando los gobiernos estatales quienes son responsables del diseño e implementación de las políticas de seguridad pública en sus jurisdicciones, promoviendo la colaboración intergubernamental con la federación y los municipios. Una consideración adicional, y que resulta un elemento fundamental del modelo ILP, es que el uso de la inteligencia permite la focalización de los recursos gubernamentales en la prevención y reducción de la criminalidad, siendo ésta una necesidad ante el reconocimiento de que la criminalidad cada vez es más compleja y los recursos gubernamentales son limitados, especialmente los destinados a la seguridad pública (Ratcliffe, 2016).

A partir de dichas consideraciones, la investigación estudia la adopción del modelo de ILP en las entidades federativas de México. Para ello, se analiza específicamente el desarrollo formal de unidades específicas para la generación de inteligencia para la seguridad pública, característica básica en la implementación del modelo ILP (OSCE, 2017; Peterson, 2003; Ratcliffe \& Guidetti, 2008), diferenciando la inteligencia para la seguridad pública, de la inteligencia policial, entendiendo a la primera como una herramienta de gestión estratégica (Carter, 2016), producto del análisis del fenómeno criminal, con el objetivo apoyar el proceso de toma de decisiones, brindando un mejor entendimiento del fenómeno criminal, identificando criminales prolíficos y peligrosos (Ratcliffe, Sorg \& Rose, 2015), contribuyendo a establecer objetivos para la contención y reducción del crimen a través de estrategias policiales y colaboración de distintas dependencias apoyándose en evidencia (Darroch \& Mazerolle, 2013); mientras que la inteligencia policial es producto de la investigación y análisis de información para la detención de criminales, así como la obtención de evidencias para lograr las sentencias judiciales contra los criminales (Chalk \& Rosenau, 
2003). La inteligencia policial y la inteligencia para la seguridad pública están relacionadas, no son excluyentes una de la otra y se retroalimentan entre sí, pero la inteligencia policial busca ser más operable, de uso más inmediato para la función policial y la inteligencia para la seguridad pública busca contribuir en la prevención, investigación y persecución de los delitos (Ratcliffe, 2016).

La diferencia entre la inteligencia policial y la inteligencia para la seguridad pública implica una diferenciación organizacional. En México, las policías son parte de las Secretarías de Seguridad Pública o Fiscalías Generales, dependencias responsables no solo de la función policial sino también de la prevención de la violencia y la delincuencia, así como de la administración penitenciaria y, en los estados en que las policías son parte de las Fiscalías, también de la investigación y persecución de los delitos; en consecuencia, el diseño e implementación de las políticas de seguridad pública es responsabilidad de las Secretarías de Seguridad o Fiscalías, las cuales son implementadas por cada una de las áreas, entre las que se encuentran las policías. Esta diferencia es fundamental, toda vez que gran parte de los estudios sobre ILP han observado Departamentos de Policía, donde las policías son responsables de la seguridad pública, pero en México, las policías son parte de la estrategia de seguridad, la cual es diseñada por el titular de la Secretaría o Fiscalía. En consecuencia, las unidades que se busca observar son áreas que cuenten con atribuciones para obtener información de todas las áreas de la dependencia responsable de la seguridad pública en cada entidad federativa y que cuente como usuario de sus productos de inteligencia al titular de la dependencia, marcando una diferencia frente a otras áreas generadoras de inteligencia policial o penitenciaria, pero que no cuentan con atribuciones para obtener información del resto de áreas de la dependencia y el usuario principal de sus productos de inteligencia es el titular de sus áreas, pero no el titular de la Secretaría o Fiscalía. Para ello, en la siguiente sección se expone la importancia de la inteligencia para la seguridad pública para, posteriormente, exponer los resultados de la investigación que se hizo al analizar la estructura de las secretarías de seguridad pública o equivalentes (SSP) en las entidades federativas, a partir de la revisión de las Leyes Orgánicas de la Administración Pública (LOAP), Leyes de Seguridad y Reglamentos Internos de las Secretarías de Seguridad Pública o equivalentes (RISSP), vigentes al mes de junio del 2020. Reconociendo que la seguridad pública es una materia concurrente entre los distintos órdenes de gobierno en México y que los fenómenos criminales trascienden los límites jurisdiccionales, el objetivo ha sido el identificar cómo las entidades federativas en México generan inteligencia para la seguridad pública, entendiendo a la seguridad pública desde un enfoque amplio, no restringido a la función policial.

El ILP es un modelo de gestión estratégica y tiene como principal objetivo el generar productos de inteligencia que contribuyan al tomador de decisiones a realizar su función de una manera estratégica, es decir, enfocando los recursos necesarios para prevenir, contener y reducir el crimen (Carter, 2016); es decir, se enfoca en la generación de productos que permitan a la dependencia la definición de prioridades, con un uso eficiente de los recursos disponibles (Ratcliffe, 2016). Frente a esta definición, el punto de partida del estudio es el reconocimiento de que las SSP cuentan con atribuciones para adoptar el ILP como modelo de gestión. Al respecto, previamente se expuso que el artículo $21^{\circ}$ Constitucional establece que la seguridad pública comprende la prevención, investigación y persecución de los delitos; en la misma dirección, el Art. 5, Fracción X de la Ley General del Sistema Nacional de Seguridad Pública reconoce a las SSP como instituciones policiales y, en consecuencia, con las atribuciones para investigar los delitos, lo cual configura, en términos generales, las 
atribuciones necesarias para la generación de inteligencia para la seguridad pública. El reconocimiento de dicho marco legal ha permitido el desarrollo de áreas de inteligencia al interior de todas las policías estatales e incluso algunas municipales, pero considerando la diferencia entre la inteligencia policial y la inteligencia para la seguridad pública, la investigación ha buscado identificar la existencia de unidades con características y atribuciones para la generación de inteligencia para la seguridad pública, reconociendo que la seguridad pública no se restringe a la función policial. Para conducir la investigación, el análisis se realizó de la siguiente manera:

- Identificación de las atribuciones para el diseño de las estrategias y políticas públicas de seguridad pública. Se revisaron todas las LOAP, con el objetivo de identificar cómo se distribuyen dichas atribuciones entre las diferentes dependencias estatales. En la misma dirección, se revisaron las leyes de seguridad pública o equivalentes para identificar los mecanismos específicos de colaboración.

- Identificación de las atribuciones específicas para la gestión y especialmente el análisis de la información de seguridad pública. Se revisaron las leyes de seguridad pública o equivalentes con el objetivo de identificar a los actores específicos responsables de la gestión y análisis de la información, así como sus atribuciones específicas para realizar el análisis y generar productos que generen conocimiento para la toma de decisiones en materia de seguridad pública en un sentido amplio, no restringido al ámbito policial.

- Análisis de las atribuciones de las áreas de análisis de la información para identificar su alcance. Se revisaron los RISSP, identificando las áreas específicas que analicen información y generen productos para la toma de decisiones, identificando su ubicación organizacional, destacando la subordinación administrativa y, a partir de identificar a su usuario principal, determinar el impacto organizacional de sus productos.

Un elemento fundamental para la investigación es el reconocimiento de la posibilidad de que, a pesar de no aparecer en el marco legal relativo a la seguridad pública en las entidades federativas, sí existan las áreas con las funciones de inteligencia; sin embargo, en caso de no aparecer en el marco legal vigente, dichas áreas serían informales, lo que las hace vulnerables a la voluntad de los titulares de las secretarías de seguridad y gobernadores de los estados, los cuales podrían debilitarlas o fortalecerlas a voluntad, pero lo más importante sería que, al no incorporarse en el marco normativo, carecerán de responsabilidades y obligaciones para la rendición de cuentas, lo cual las hace susceptibles de convertirse en áreas de espionaje, además de carecer de mecanismos normativos que permitan comprometer recursos públicos para su ejercicio.

\section{INTELIGENCIA PARA LA SEGURIDAD PÚBLICA}

El punto de partida de la investigación ha sido la definición del modelo de Acción Policial Guiada por Inteligencia o Intelligence-Led Policing como un modelo de gestión estratégica, que tiene como objetivo central generar productos de inteligencia que contribuyan a los procesos de toma de decisiones en materia de seguridad pública, contribuyendo a la 
prevención, contención y reducción de los delitos, a través de estrategias basadas en evidencia (Carter, 2016; Ratcliffe, 2016).

El modelo fue considerado inicialmente como el desarrollo de capacidades por parte de las instituciones policiales para la investigación de los delitos (Treverton, 2011); sin embargo, el ILP no se refiere únicamente al ejercicio de la función policial sino a un modelo de gestión y se enfoca en el proceso de toma de decisiones. El modelo nació en el Reino Unido, en la década de los 90s, pero tuvo su mayor impulso después de los ataques terroristas del 9/11 en los EE. UU., cuando fue reconocido como una herramienta para la seguridad interior al articular a las policías estatales como proveedoras de información para el contraterrorismo (Chlak \& Rosenau, 2003), pero ha sido implementado con resultados positivos en ciudades como Los Ángeles (Tita et al., 2003), Nueva York (Reppetto, 2011), Nueva Jersey (Ratcliffe, 2016), Denver (Massarotti, 2012), Santiago de Chile (Tudela, 2012), entre muchos otros.

A pesar de ser reconocido como un instrumento para la seguridad interior, el ILP rápidamente demostró su capacidad para cambiar los paradigmas en la organización, definición de objetivos y tácticas de las policías, fortaleciendo la transformación desde un enfoque principalmente reactivo hacia uno proactivo, preventivo y focalizado (Darroch \& Mazerolle, 2013; Ratcliffe, 2016). La transformación de las policías expuso que la inteligencia no solo es útil para la acción policial sino también para la definición de tácticas y estrategias para la seguridad, atribución ya no de los policías sino de los tomadores de decisiones dentro de la organización responsable de la seguridad (Carter, 2016), por lo que el modelo no es operativo sino que es más bien de gestión y busca que la toma de decisiones sea estratégica, orientada al futuro, focalizada en delitos y criminales específicos, orientada hacia la prevención y reducción del crimen (Burcher \& Whelan, 2019).

La implementación del ILP implica una transformación organizacional dentro de las dependencias responsables de la seguridad. Al respecto, numerosos estudios han expuesto que dicha transformación no es sencilla ya que implica transformaciones en la estructura organizacional (Ratcliffe \& Guidetti, 2008); en las herramientas de gestión y administración, así como los procesos para la hechura y toma de decisiones; y en la propia cultura de la organización (Carter \& Carter, 2009; Darroch \& Mazerolle, 2013). Frente a dichos cambios, se encuentran las críticas que señalan al ILP como un método tecnocrático, reservado a instituciones grandes, con recursos y personal suficiente para el desarrollo de las unidades necesarias para impulsar el modelo (Carter \& Carter, 2009); que ha abierto la puerta a la violación de ciertos derechos civiles, permitiendo la sobre-vigilancia de los ciudadanos bajo el argumento de sospechas de terrorismo o participación en actividades delictivas (Carter \& Carter, 2009; Carter, Carter \& McGarrell, 2017); y que carece de suficiente evidencia que evalúe su desempeño y demuestre su efectividad (James, 2003).

La adopción del modelo ILP implica una transformación en el liderazgo, la cultura organizacional al interior de la dependencia, el establecimiento de lazos con otras dependencias para el intercambio de información, entre otros. Por un lado, el tomador de decisiones requiere de acercarse con el analista de inteligencia para la definición de objetivos y la adecuada difusión de los productos de inteligencia (OSCE, 2017). Por otro lado, los analistas deben reconocer su papel en el diseño de estrategias de seguridad pública y para la función policial, reorientando su trabajo desde un enfoque tradicionalmente enfocado hacia la operación, a que los productos sean operables, hacia la prevención y el diseño de 
estrategias de seguridad, más allá de la acción policial (Carter \& Phillips, 2015). Finalmente, el cambio en la cultura organizacional implica difundir entre la organización responsable de la seguridad pública su papel como proveedores de insumos, ya sea información o productos de inteligencia de sus áreas, así como consumidores de los productos de inteligencia para la seguridad, a través de las estrategias impulsadas por el tomador de decisiones (Ratcliffe \& Guidetti, 2008); sin embargo, este cambio generalmente enfrenta intereses políticos al interior de las organizaciones e implica procesos burocráticos que hacen más lento el proceso de adopción (Carter, 2009).

La principal fortaleza de la ILP es el fortalecimiento a los procesos de toma de decisiones, permitiendo el uso eficiente de los recursos disponibles. Por un lado, ha demostrado su utilidad en el fortalecimiento de las políticas de seguridad interior contra el terrorismo (Chalk \& Rosenau, 2003), pero también frente a retos complejos como crimen organizado y grandes pandillas (Ratcliffe, 2016; Ratcliffe, Sorg \& Rose, 2015). En este punto, el modelo resulta muy atractivo para el caso mexicano, toda vez que los estados enfrentan como principal reto al crimen organizado que es un fenómeno muy complejo, con recursos humanos y financieros muy restringidos.

La inteligencia para la seguridad pública constituye el insumo para el ILP. Ésta, a su vez es producto de un conjunto de procesos que se articulan en lo que se ha denominado como "ciclo de inteligencia", el cual es un conjunto de procesos, los cuales varían en número de acuerdo con los distintos autores que lo han desarrollado, pero que en términos generales implica el análisis de la información, a través de herramientas científicas y tecnológicas, para la generación de conocimiento operable para el proceso de toma de decisiones (Esteban y Navarro, 2003; Herrera, 2009; Navarro, 2004; Tello, 2012). En términos organizacionales, la inteligencia se produce en unidades o áreas específicas especializadas, empleando el ciclo inteligencia como guía para la conducción de sus procesos. Si bien el ciclo de inteligencia tiende a estructurar el área productora de inteligencia, esto no significa que el proceso de generación de inteligencia sea rigurosamente unidireccional. En cambio, la generación de inteligencia es mucho más fluida, no sigue los pasos de manera rigurosa debido a contextos de urgencia o necesidades específicas tanto de los analistas como de los tomadores de decisiones (Burcher \& Whelan, 2019).

La ubicación del área productora de inteligencia es determinante para el modelo ILP. Al señalar que el ILP es un modelo de gestión estratégica, que contribuye al proceso de toma de decisiones para la seguridad pública, la ubicación de las áreas determina sus atribuciones, sus límites y su subordinación administrativa. En este sentido, para que la inteligencia que se genera cumpla con su función estratégica, es necesario que se encuentre directamente subordinada al tomador de decisiones para el diseño de la estrategia de seguridad pública (Chalk \& Rosenau, 2003; Johnson, 2003; Liebowitz, 2010; Ratcliffe \& Guidetti, 2008). De no ser así, en caso de que el área se encuentre subordinada a otra área, sus atribuciones igualmente se encontrarán limitadas por su ubicación administrativa y su rol estratégico como soporte de la toma de decisiones basadas en evidencia estará restringido. Adicional a la existencia de un área específica productora de inteligencia, es ideal el cambio en la cultura organizacional de la dependencia responsable de la seguridad pública para la constitución de una comunidad de inteligencia al interior de la propia dependencia para la compartimentación de productos de inteligencia (Ratcliffe \& Guidetti, 2008). 
A partir de las características del modelo ILP, en el siguiente apartado se busca identificar la forma como las entidades federativas de México generan inteligencia para la seguridad pública, identificando dichas áreas, con las características previamente comentadas, destacando su ubicación organizacional, es decir, que cuenten con atribuciones para la generación de inteligencia y que estén directamente subordinadas al tomador de decisiones en materia de seguridad pública, entendiendo a la seguridad como una materia que implica la prevención, investigación, persecusión y sanción de los delitos, labores que trascienden a la función policial.

\section{INTELIGENCIA PARA LA SEGURIDAD PÚBLICA EN LAS ENTIDADES FEDERATIVAS EN MÉXICO}

Ante la complejidad de la distribución de atribuciones en materia de seguridad pública en México, el objetivo de este apartado es identificar las responsabilidades específicas al interior de los gobiernos de los estados para el diseño de las estrategias de seguridad pública. Al respecto, la seguridad pública en las entidades federativas se estructura, en general, en tres etapas, en las que participan diferentes dependencias con atribuciones específicas en cada una de ellas:

I. Prevención, primera respuesta e investigación (primeros actos ante un evento flagrante), los cuales generalmente corresponden a las SSP ${ }^{1}$, implican de manera central a la función policial pero también políticas públicas para la prevención de la violencia y la delincuencia.

II. Investigación y persecución de los delitos, los cuales corresponden a las Fiscalías de Justicia, excepto en el caso de Chihuahua, donde la Fiscalía General es responsable tanto de la prevención, como la investigación y persecución de los delitos (Ley Orgánica de la Fiscalía General del Estado de Chihuahua, 2010, Artículo 1) y para lo cual se apoyan del concepto de inteligencia criminal, la cual se refiere al uso de la inteligencia para el estudio del crimen y de los criminales (ONUDC, 2011), así como la persecución de los delincuentes para la procuración de justicia (Torres y Santiago, 2015).

III. Sanción de los delitos y las faltas administrativas, las cuales implican la administración de los sistemas penitenciarios, atribución que en ocasiones corresponde a la Secretaría de Gobierno o la SSP.

Las Secretarías de Gobierno y las SSP forman parte de la administración pública centralizada y están subordinadas administrativamente al gobernador del estado; sin embargo, las Fiscalías son organismos autónomos, no subordinados al gobernador y, en consecuencia, se requieren mecanismos para la coordinación entre las diferentes dependencias. Además de los dilemas de coordinación al interior del ejecutivo estatal, debe recordarse la concurrencia de los municipios en materia de seguridad pública, enfatizando que los municipios en México son autónomos.

\footnotetext{
${ }^{1}$ La mayoría de las entidades federativas cuentan con Secretarías de Seguridad Pública, equivalentes a un ministerio estatal, salvo en los estados de Morelos (LOAP Morelos, 2019, Artículo 33), donde la figura es una Comisión Estatal de Seguridad adscrita a la oficina del gobernador; y el estado de Tlaxcala, donde también es una Comisión, pero adscrita a la Secretaría General de Gobierno (LOAP Tlaxcala, 2015, Artículo 2).
} 
La coordinación para la seguridad en las entidades federativas se ha hecho a partir de reproducir la estructura de coordinación nacional, considerando en sus respectivas leyes de seguridad la constitución de Sistemas de Seguridad Pública, Consejos de Coordinación de los Sistemas y la figura de Secretariados Ejecutivos de los Consejos. Esta situación, implica la participación de tres actores en la definición de estrategias de seguridad pública: las SSP, responsables de la política de seguridad pública; las Fiscalías Generales de Justicia, responsables de la política de procuración de justicia y con incidencia en la política de seguridad pública; y los Secretariados Ejecutivos, responsables de la coordinación intergubernamental y que generalmente son parte de las Secretarías Generales de Gobierno ${ }^{2}$.

La investigación se enfoca en la función de las SSP, las cuales se involucran en la prevención de la violencia y la delincuencia, la primera respuesta, apoya la investigación de los delitos y, en la mayoría de los estados, es responsable de la administración penitenciaria. En consecuencia, cuando se habla de inteligencia para la seguridad pública entendemos la generación de conocimiento, a través del análisis de la información de seguridad pública, para el diseño de estrategias y políticas públicas que incidan en la seguridad pública, entendiendo a la materia más allá de la función policial, involucrando también la prevención y la administración penitenciaria. En consecuencia, se buscará identificar áreas generadoras de inteligencia o que analicen la información de seguridad pública, para apoyar los procesos de toma de decisiones de las SSP, no solo de áreas específicas dentro de las dependencias. A continuación, se expondrán los resultados del estudio, identificando en primer lugar las áreas gestoras de la información y posteriormente las áreas con atribuciones para analizar la información o para la generación de inteligencia estratégica.

\section{GESTIÓN Y ANÁLISIS DE LA INFORMACIÓN DE SEGURIDAD}

La gestión de la información en materia de seguridad pública se contempla en el artículo 21, décimo párrafo, inciso b), de la CPEUM, en el que se establece la existencia del sistema nacional de información que debe ser administrado por la federación, pero contemplando la participación de las entidades federativas y municipales para su integración y uso ${ }^{3}$ (Acosta y Chávez, 2012). Adicionalmente, a partir del 2008 todas las policías cuentan con atribuciones para la investigación de los delitos y, a partir de la armonización legislativa en las entidades federativas, se encuentra en la normatividad las atribuciones para la realización de labores de investigación, análisis de la información e inteligencia, aunque las policías estatales han avanzado lentamente en el desarrollo de estas capacidades (Moloeznik y Balcázar, 2013).

La generación de inteligencia en las entidades federativas comenzó a ser impulsada por la administración del gobierno federal 2012-2018, a través de la Estrategia 5.5 del Programa Nacional de Seguridad Pública 2014-2018, consistente en el fomento y fortalecimiento de

\footnotetext{
${ }^{2}$ En la mayoría de las entidades el Secretariado Ejecutivo se deposita en órganos desconcentrados o unidades administrativas de las Secretarías de Gobierno, salvo en los casos de Baja California, Guerrero, Querétaro, Michoacán, Sinaloa, Sonora y Veracruz, que depositan el Secretariado dentro de las Secretarías de Seguridad, así como Chihuahua, ciudad de México y Nayarit, que lo depositan en las Fiscalías.

${ }^{3}$ La colaboración intergubernamental para el análisis de la información se planteó desde el desarrollo de la Plataforma México hoy Centro Nacional de Información; sin embargo, los controles para tratar de evitar la fuga de información hicieron muy restrictivo el acceso a la plataforma por parte de funcionarios de los gobiernos estatales y municipales, lo que se ha traducido en que dicha plataforma, que ha evolucionado en el Centro Nacional de Información, en realidad sea inoperante (Sheremetov, 2012).
} 
los sistemas de intercambio de información entre los tres órdenes de gobierno, así como por el Acuerdo 08/XXXVI/2014 del Consejo Nacional de Seguridad Pública, en que los gobernadores y Jefe de Gobierno de la Ciudad de México se comprometieron a desarrollar sus correspondientes áreas de inteligencia, lo cual se reiteró en el Acuerdo 18/XL16 del mismo Consejo. Adicionalmente, la Comisión Nacional de Seguridad, hoy Secretaría de Seguridad y Protección Ciudadana, informó la constitución de la red nacional de analistas, orientado a la implementación de políticas policiales basadas en evidencia, dejando de lado el reconocimiento de que la seguridad es más que la acción policial (Gobernación, 2017). En la misma dirección, el Plan Nacional de Desarrollo 2019-2024 plantea como uno de sus objetivos la constitución de un Sistema Nacional de Inteligencia (Gobierno de México, 2019), sin detallar las acciones específicas a trabajar con las entidades federativas.

El estigma asociado al concepto de inteligencia dificulta encontrarlo en la normatividad en materia de seguridad pública. En este sentido, únicamente los estados de Campeche (LOAP Campeche, 2019, Artículo 38, F. IX), Colima (LOAP Colima, 2018, Artículo 35, F. VI), Jalisco (LOAP Jalisco, 2018, Artículo 31, F. 20), México, Nuevo León y Puebla (LOAP Puebla, 2019, Artículo 46, F. XXXV) incorporan explícitamente la generación y uso de inteligencia como insumo para la toma de decisiones en materia de seguridad pública, ya sea en las Secretarías de Gobierno (México [LOAP Estado de México, 2020, Artículo 21 bis, F. VIII], Nuevo León [LOAP Nuevo León, 2020, Artículo 20, F. X]), Finanzas (Colima [LOAP Colima, Artículo, 23, F. XXIX], Puebla [LOAP Puebla, 2019, Artículo 33, F. LVIII] y SSP. Frente a estos casos, destacan Chiapas (RISSP Chiapas, 2018, Artículo 21, F. XIV) y la Ciudad de México (RISSP Ciudad de México, 2020, Artículo 46, F. XIII) que, en sus respectivos RISSP, contemplan explícitamente la generación de inteligencia estratégica.

De acuerdo con las leyes de seguridad pública, la información de seguridad se gestiona a través de Sistemas Estatales de Información de Seguridad Pública. La mayoría de las entidades depositan las atribuciones para la gestión de dichos sistemas en las Secretarías de Seguridad y, específicamente en los Centros de Coordinación Integral (C5). A partir de la revisión de las LOAP, las leyes de seguridad y los RISSP en las entidades federativas se identificaron las siguientes áreas gestoras de información de seguridad pública:

Cuadro 1. Áreas gestoras de información de seguridad pública

\begin{tabular}{|c|l|l|}
\hline & \multicolumn{1}{|c|}{$\begin{array}{c}\text { Secretaría de Seguridad Pública o } \\
\text { equivalente }\end{array}$} & $\begin{array}{c}\text { Secretariados Ejecutivos de Consejos } \\
\text { de Seguridad }\end{array}$ \\
\hline Aguascalientes & Policía Estatal & \\
\hline Baja California & Coordinación de Estadística & \\
\hline Baja California Sur & Dirección del C4 & \\
\hline Campeche & Unidad de Análisis & \\
\hline Chihuahua & $\begin{array}{l}\text { Departamento de Estadística Delictiva } \\
\text { Procesal y Judicial (Fiscalía) }\end{array}$ & \\
\hline Coahuila & Dirección de Sistemas de Información & \\
\hline Colima & Policía Estatal & \\
\hline Chiapas & $\begin{array}{l}\text { Unidad de Tecnologías y Gestión de la } \\
\text { Información Ejecutiva de Análisis e }\end{array}$ & \\
\hline Ciudad de México & $\begin{array}{l}\text { Dirección } \\
\text { Inteligencia Policial }\end{array}$ \\
\hline
\end{tabular}




\begin{tabular}{|c|c|c|}
\hline Durango & Unidad de Enlace Informático & \\
\hline Guerrero & & $\begin{array}{l}\text { Dirección General del Sistema Estatal } \\
\text { de Información Policial }\end{array}$ \\
\hline Guanajuato & Dirección de registro y base de datos & \\
\hline Hidalgo & $\begin{array}{l}\text { Subsecretaría de Prevención y Coordinación } \\
\text { Institucional }\end{array}$ & \\
\hline Jalisco & $\begin{array}{l}\text { Centro de Inteligencia y Comunicaciones } \\
\text { para la Seguridad }\end{array}$ & \\
\hline Estado de México & Centro de Mando y Comunicación & Centro de Información y Estadística \\
\hline Michoacán & Unidad de Análisis e Inteligencia Estatal & \\
\hline Morelos & Centro Estatal de Información & \\
\hline Nayarit & & $\begin{array}{l}\text { Dirección General del Centro Estatal } \\
\text { de Información }\end{array}$ \\
\hline Nuevo León & Dirección de Servicios del C5/Policía Estatal & \\
\hline Oaxaca & $\begin{array}{l}\text { Subsecretaría de Información y Desarrollo } \\
\text { Institucional }\end{array}$ & \\
\hline Puebla & Dirección del C4 & \\
\hline Querétaro & Dirección de prevención/Policía Estatal & \\
\hline Quintana Roo & $\begin{array}{|lll|}\begin{array}{l}\text { Departamento de análisis estadístico y } \\
\text { análisis informático }\end{array} & \\
\end{array}$ & \\
\hline San Luis Potosí & & Consejo Estatal de Seguridad Pública \\
\hline Sinaloa & & $\begin{array}{l}\text { Dirección General del Centro Estatal } \\
\text { de Información }\end{array}$ \\
\hline Sonora & $\begin{array}{l}\text { Coordinación General del Sistema Estatal de } \\
\text { Información }\end{array}$ & \\
\hline Tabasco & Policía Estatal & \\
\hline Tamaulipas & Planeación y Estadística & \\
\hline Tlaxcala & & $\begin{array}{l}\text { Dirección General del Centro Estatal } \\
\text { de Información }\end{array}$ \\
\hline Veracruz & Subdirección de estadística delictiva & $\begin{array}{l}\text { Coordinación del Sistema Estatal de } \\
\text { Información }\end{array}$ \\
\hline Yucatán & $\begin{array}{l}\text { Unidad de Análisis de Información e } \\
\text { Inteligencia }\end{array}$ & \\
\hline Zacatecas & Policía Estatal & Centro Estatal de Información \\
\hline
\end{tabular}

Fuente: Elaboración propia

A partir del estudio de las atribuciones de cada una de estas áreas se identificó que, a pesar de ser responsables de la gestión de la información, no cuentan con atribuciones explícitas para su análisis y, quienes lo hacen, lo restringen a la generación de información para el ejercicio de las funciones policiales, sin considerar otros elementos vinculados a la seguridad pública como la prevención de la violencia y la delincuencia ${ }^{4}$. A partir de la revisión de las LOAP y las Leyes de Seguridad, se identificó la siguiente distribución de atribuciones para el análisis de la información de seguridad:

\footnotetext{
${ }^{4}$ Adicionalmente, algunos estados incorporan en el nombre de sus Centros de Coordinación, la función de Inteligencia, como en los casos de Guanajuato, Hidalgo o Sonora, pero el RISSP no brinda a dicho Centro de atribuciones para la generación de inteligencia, ni tampoco para el análisis de la información de seguridad pública.
} 
Cuadro 2. Dependencias con atribuciones explícitas para el análisis de información de seguridad pública

\begin{tabular}{|c|c|c|c|}
\hline $\begin{array}{c}\text { No establece } \\
\text { explícitamente } \\
\text { las atribuciones }\end{array}$ & Fiscalías & $\begin{array}{c}\text { Secretariados } \\
\text { Ejecutivos }\end{array}$ & $\begin{array}{c}\text { Secretarías de } \\
\text { Seguridad }\end{array}$ \\
\hline Baja California & Campeche & Guanajuato & Aguascalientes \\
\hline $\begin{array}{c}\text { Baja California } \\
\text { Sur }\end{array}$ & Chihuahua & Jalisco & Chiapas \\
\hline Hidalgo & Durango & Nuevo León & Coahuila \\
\hline & Tabasco & Querétaro & $\begin{array}{c}\text { Ciudad de } \\
\text { México }\end{array}$ \\
\hline Sinaloa & & Veracruz & Colima \\
\hline Yucatán & & & Durango \\
\hline & & & $\begin{array}{c}\text { Estado de } \\
\text { México }\end{array}$ \\
\hline & & & Guerrero \\
\hline & & & Michoacán \\
\hline & & & Morelos \\
\hline & & & Nayarit \\
\hline & & & Oaxaca \\
\hline & & & Puebla \\
\hline & & & Quintana Roo \\
\hline & & & Sanoras \\
\hline
\end{tabular}

Fuente: Elaboración propia con base en las LOAP y Leyes de Seguridad

Es importante reiterar que el hecho de que la normatividad no establezca explícitamente las atribuciones para el análisis de la información de seguridad pública para la generación de inteligencia no significa que las SSP no realicen estas labores. Sin embargo, el hecho de que no se contemple en la normatividad implicaría que dichas actividades se realizan de manera informal y, en consecuencia, sin protocolos, recursos, ni controles claros.

La normatividad no es clara en torno a los objetivos del análisis de la información de seguridad pública. Estados como Aguascalientes, Morelos, Guanajuato, Sonora y Baja California señalan que el objetivo del análisis es el estudio de la incidencia criminológica; Coahuila, Colima y Sinaloa, orientan el análisis hacia la problemática de prevención, procuración de justicia y seguridad; mientras que Veracruz señala que el objetivo es la 
obtención, procesamiento, explotación y análisis de la información de seguridad pública, mientras que el resto de los estados no establecen algún objetivo para el análisis. En este sentido, la inteligencia en materia de seguridad pública no tiene un objetivo homogéneo entre las entidades federativas y solo en los estados de Chiapas, Ciudad de México, Estado de México, Hidalgo, Nuevo León se contempla la orientación de la inteligencia para la seguridad pública contemplando no solo la acción policial sino también la prevención, la procuración de justicia y seguridad.

Si bien son pocas las entidades que consideran la importancia de la inteligencia para la seguridad pública son menos las que consideran la integración de las atribuciones de análisis de la información en áreas estratégicas. Para ello, la investigación buscó áreas con al menos dos características derivadas de la definición de inteligencia estratégica: a) que tuvieran atribuciones para el análisis de la información, y b) que su ubicación orgánica y nivel de mando estuviera supeditada directamente al titular de la secretaría o equivalente, tal que cuente con atribuciones para obtener información de todas las áreas de la dependencia, reciba requerimientos y genere productos directamente para el titular. Al respecto, destacan las siguientes áreas con atribuciones para la generación de inteligencia estratégica en materia de seguridad pública 5 :

- Aguascalientes. Dirección General de Análisis e Inteligencia, la cual depende orgánicamente de la Subsecretaría de Seguridad Pública, la cual, a su vez, depende directamente del Secretario de Seguridad Pública. Cuenta con tres Departamentos, uno de ellos enfocado en Inteligencia, otro para Análisis y el último para Planeación; sus atribuciones se enfocan en el estudio de los delitos y los delincuentes, enfatizando el enfoque policial; sin embargo, también considera su participación en la planeación, prospectiva, evaluación, control de objetivos y metas de la Secretaría (RISSP Aguascalientes, 2017, Artículos 22-24). El área puede considerarse de inteligencia estratégica, toda vez que sus productos impactan a la Secretaría, pero sus atribuciones se restringen a la función policial.

- Baja California. Dirección de Información, Análisis y Estrategias, la cual depende directamente del titular de la Secretaría de Seguridad Pública, por lo que sí se ubica en una posición estratégica. Las atribuciones de esta dependencia se relacionan con la gestión de la secretaría, por lo que se le puede considerar un área estratégica. Entre sus facultades, destacan la coordinación de la estrategia policial de prevención del delito, la coordinación de las estrategias policiales, el acceso a los bancos institucionales de datos, realizar análisis para disminuir el fenómeno delictivo, proponer políticas para combatir el fenómeno criminal, entre otras (RISSP Baja California, 2010, Artículo 64).

- Campeche. En este caso la Policía Estatal cuenta con atribuciones para la generación de inteligencia para la prevención de delitos, pero restringido a sus atribuciones, por lo que no puede integrar información del resto de la secretaría; en cambio, la Unidad de análisis y estadística cuenta con atribuciones explícitas para la generación de inteligencia y forma parte de la Oficina del Secretario, por lo que también se constituye como un área estratégica (RISSP Campeche, 2018, Artículo 39).

\footnotetext{
${ }^{5}$ En la siguiente lista se ha excluido el caso de Tlaxcala, cuya SSP cuenta con una Dirección de Inteligencia; sin embargo, a la fecha de elaboración del presente, aún no se concluía el proceso de armonización normativa.
} 
- Chihuahua. Dirección de la División de Inteligencia, dependiente de la Dirección General Operativa, la cual a su vez depende de la Comisión Estatal de Seguridad y la cual forma parte de la Fiscalía General del Estado. Entre sus atribuciones, destaca la dirección de acciones en materia de inteligencia con el fin de evitar y disminuir la comisión de delitos, gestión de la información en materia de seguridad pública para la adopción de estrategias en materia de seguridad pública, detectar los factores de amenazas o riesgos que atenten contra la preservación de las libertades, el orden y la paz públicas, informar a la persona titular de la comisión las agendas de riesgo que se deriven del análisis y valoración de la información, entre otras (Reglamento Interior de la Fiscalía General de Chihuahua, 2018, Artículo 119).

- Chiapas. En este caso, la oficina del secretario cuenta con la Unidad de Inteligencia, la cual, genera inteligencia estratégica, especialmente para apoyar las funciones del Estado Mayor, pero también genera inteligencia directamente para el Secretario, lo que la constituye como un área de inteligencia estratégica (RISSP Chiapas, 2018, Artículos 21 y 30).

- Ciudad de México. En este caso se identificaron diferentes áreas relacionadas con la investigación, análisis e inteligencia. En primer lugar, cuenta con una Subsecretaría de Información e Inteligencia Policial. Adicionalmente, cuenta con una Subdirección de Información, perteneciente a la Dirección Ejecutiva de Comunicación Social y de la cual se desprende la Unidad Departamental de Análisis de la Información. En tercer lugar, dentro de la Dirección General de Asuntos Internos cuenta con una Dirección de Investigación Policial. En cuarto lugar, encontramos la Subdirección de Análisis y Estudios para la prevención del delito. El área que realiza las principales actividades relacionadas con el análisis e inteligencia es la Subsecretaría de Información e Inteligencia Policial. Destaca que la Dirección General de tecnologías de Información y Comunicaciones es la proveedora de información. Entre las atribuciones de la subsecretaría destaca en primer lugar su relación directa con el secretario para el desarrollo tecnológico de la secretaría, así como para la toma de decisiones que promuevan la seguridad, por lo que su actividad se relaciona estratégicamente con la secretaría. Destacan también, el fortalecer la función policial con información estadística que brinde los elementos para la toma de decisiones que promuevan la seguridad de la ciudadanía, entre otras (RISSP Ciudad de México, 2020, Artículo 14).

- Colima. La SSP cuenta con una Coordinación General de Información, la cual gestiona las plataformas de información de seguridad, y de la cual se desprende la Coordinación de Análisis de Información, que únicamente tiene res atribuciones: proponer lineamientos para el análisis, analizar la información, proponer mecanismos de resguardo de la información (RISSP Colima, 2017, Artículos 17-20).

- Durango. En este caso encontramos a la Dirección de Análisis de la Información, la cual tiene entre sus atribuciones la generación de productos para la toma de decisiones de la secretaría, destacando entre sus atribuciones la sistematización, consulta, análisis y actualización de la información que diariamente se genere sobre seguridad pública para la toma de decisiones (RISSP Durango, 2011, Artículo 30); elaborar las agendas de riesgo para el titular de la dependencia, entre las más destacadas; sin embargo, la generación de productos directamente para el Secretario contrasta con su ubicación orgánica, toda vez que depende directamente de la Subsecretaría Operativa, 
por lo que no necesariamente podrá obtener la información de áreas en su mismo nivel organizacional y superiores dentro de la Secretaría. En consecuencia, el área tiene funciones estratégicas, pero su ubicación orgánica limita su alcance.

- Estado de México. El RISSP brindan al secretario atribuciones para la generación de inteligencia para la prevención y combate a los delitos, las cuales se distribuyen entre la Dirección General de Información (DGI) y la Unidad de Inteligencia e Investigación para la Prevención, la cual depende de dicha dirección (RISSP Estado de México, 2020, Artículos 14 F. XXXIX y 35). La Unidad cuenta con una compleja estructura, integrada por una Dirección de Inteligencia, una de Investigación y una tercera de Enlace Táctico; a partir de la revisión de sus atribuciones destaca el análisis de los fenómenos delincuenciales, el estudio de los grupos delictivos. De manera complementaria, la DGI cuenta con la responsabilidad de brindar información útil para combatir el delito y fortalecer la justicia, para lo cual es responsable de articular un conjunto de bases de datos con información delictiva y de seguridad, proporcionada por todas las unidades administrativas de la secretaría, lo que la convierte también en un área de inteligencia estratégica. En consecuencia, se puede señalar que el Estado de México cuenta con una de las áreas de inteligencia estratégica con mayores atribuciones y con una de las estructuras más complejas.

- Jalisco. Jefatura de Inteligencia. En esta entidad, la Fiscalía General cuenta con un Centro de Inteligencia y Comunicaciones para la Seguridad y de manera específica también una Comisaría de Seguridad Pública, la cual a su vez cuenta con un comisario Jefe de Inteligencia. La Dirección del Centro de Inteligencia responde directamente al Fiscal General y se apoya para su operación de tres direcciones: Política Criminal y Estadística, Criminalística y Centro Integral de Comunicaciones. Entre sus facultades, se encuentran Implementar y Operar el Sistema Estatal de Inteligencia y Comunicaciones para la Seguridad, Proponer estrategias para la aplicación ágil y eficiente de las políticas públicas derivadas de labores de inteligencia. En pocas palabras, el Centro se convierte en un nodo de información estratégica para el diseño de las políticas en materia de seguridad en Jalisco (Reglamento Interno de las Instancias Administrativas del Despacho del Fiscal General, 2014, Artículo 22).

- Morelos. A diferencia del resto de los estados, las atribuciones de seguridad pública se ejercen directamente por el gobernador del estado, a través de una Comisión Estatal de Seguridad; a su vez, dicha Comisión cuenta con un Centro de Análisis de Información sobre Seguridad Pública, el cual es responsable de la gestión de información de seguridad pública y tiene la obligación de proveer información a todas las áreas, analizar la información generada por todas las áreas de la Comisión y canalizar sus productos directamente al Comisionado, por lo que se puede identificar claramente como un área de inteligencia estratégica (RISSP Morelos, 2017, Artículo 53).

- Puebla. Subsecretaría de Inteligencia e Investigación, dependiente del Secretario de Seguridad Pública. En esta entidad se cuenta con una Subsecretaría de Inteligencia e Investigación, la cual se apoya de tres direcciones generales, la de Inteligencia e Investigación, la de Análisis Criminal y la de Servicios Técnicos, tal como se expone a continuación: Entre las facultades de la subsecretaría, de acuerdo al artículo 31 del RISSP, se establecen: Analizar los fenómenos delincuenciales, con la finalidad de 
generar productos de inteligencia que permitan instrumentar estrategias orientadas a la desarticulación de las bandas delictivas, Implementar las acciones necesarias destinadas a la obtención de información, Establecer, operar y actualizar los sistemas de información estadística y de análisis necesarios para la generación de productos de inteligencia encaminados a desalentar conductas ilícitas, Dictar políticas y estrategias de acción para optimizar el proceso de acopio y análisis de información referente a la identificación y evolución del fenómeno delincuencial en el Estado. En otras palabras, el estado de Puebla cuenta con una de las áreas de inteligencia más complejas de México.

- Querétaro. La SSP del estado cuenta con una Unidad de Análisis e Información, con atribuciones para integrar, analizar y procesar la información en materia de seguridad, a través de distintas metodologías para la generación de productos que contribuyan a una política de seguridad integral (RISSP Querétaro, 2020, Artículo 44bis); si bien dichas atribuciones la caracterizan como un área generadora de inteligencia estratégica, orgánicamente depende de la Dirección de Servicios al Público y Seguridad Privada, la cual a su vez forma parte de la Subsecretaría de Policía Estatal, por lo que en realidad constituye un área de tercer nivel y sus atribuciones están limitadas por la subsecretaría y, en consecuencia, no podrá ni allegarse de información del resto de la secretaría, ni generará productos directamente para el secretario; en consecuencia, no es un área de inteligencia estratégica.

- Tamaulipas. La SSP cuenta con una Dirección de Análisis e Inteligencia, dependiente de la Subsecretaría de Operación Policial; sin embargo, sus atribuciones son muy escuetas, y no atribuye claramente las funciones de análisis, ni generación de productos de inteligencia. En consecuencia, a pesar de llamar la atención por su denominación, no cuenta con atribuciones claras para la generación de inteligencia y tiene una ubicación organización que la limita a las atribuciones de la subsecretaría (RISSP Tamaulipas, 2013, Artículo 12).

El estudio muestra que menos de la mitad de los estados han desarrollado áreas con atribuciones explícitas para el análisis de información de seguridad pública o la generación de inteligencia estratégica, es decir, dirigida a contribuir al proceso de toma de decisiones alrededor de la seguridad pública desde una visión amplia, no restringida al ámbito policial. A pesar de ello, no hay un modelo homogéneo y algunos estados, como Aguascalientes, Durango, Querétaro o Tamaulipas, cuentan con áreas generadoras de inteligencia, pero las ubican organizacionalmente en una posición que no les permite contar con una comunicación directa con el titular de la SSP, por lo que no permite que sus productos tengan un impacto estratégico. Adicionalmente, debe recordarse que, en la mayoría de los estados, las áreas gestoras de la información, no cuentan con atribuciones para analizarla, lo cual implica el no aprovechar el potencial de estas áreas. Finalmente, también se tiene presente que, el hecho de que el análisis y la generación de inteligencia estratégica no se encuentren en la normatividad de las entidades, no significa que no se realicen estas acciones, pero en caso de realizarse fuera del marco normativo, implica su carencia de legalidad, su falta de institucionalidad y, en consecuencia, se ubican en una posición endeble frente a cambios e intenciones políticas. 


\section{CONCLUSIONES}

La inteligencia para la seguridad pública es una herramienta de gestión estratégica que fortalece el proceso de toma de decisiones con base en evidencia, permitiendo el diseño de estrategias para prevenir, contener y reducir el crimen, focalizando los recursos disponibles de una manera más eficiente (Ratcliffe, 2016). La adopción del modelo ILP implica cambios en la forma de ejercer el liderazgo, la estructura y cultura organizacional, los cuales no son sencillos, ni rápidos, reconociendo que los cambios en estructuras gubernamentales y, sobre todo en instituciones de seguridad son complejas ya que son instituciones con jerarquías rigurosas y una cultura organizacional particular (Darroch \& Mazerolle, 2013; Ratcliffe \& Guidetti, 2008).

En México, la seguridad es una materia que requiere de una gobernanza multinivel, con la participación de los gobiernos municipales, estatales y federal. Los gobiernos tienen el reto de coordinarse entre ellos, pero también en su interior con las diferentes dependencias con atribuciones para la prevención, investigación, persecución y sanción de los delitos. En este sentido, la investigación se propuso como objetivo estudiar la adopción del modelo ILP en las entidades federativas, a partir de observar un elemento básico del modelo: la existencia de áreas especializadas en la producción de inteligencia para la seguridad pública, marcando una clara diferencia entre la inteligencia para la seguridad de la inteligencia policial.

La investigación ha arrojado como resultado que la mayoría de las entidades federativas carecen de áreas generadoras de inteligencia para la seguridad pública, con atribuciones para integrar la información que se genera en distintas áreas de las SSP y generar productos que apoyen el proceso de toma de decisiones para la seguridad pública de manera estratégica, basándose en evidencia. Esta afirmación no significa que los titulares de las SSP no utilicen inteligencia, ni evidencia para su toma de decisiones, pero las SSP carecen de áreas que generen productos directamente para los titulares, lo que puede derivar en información sin analizar y dispersa. La comprobación de la forma como se gestiona la información en cada una de las entidades requiere de investigaciones posteriores que estudien cada caso, empleando diferentes herramientas para la investigación y permitan identificar con mayor detalle la forma de ejercer el liderazgo y la cultura organizacional.

Frente a la complejidad de la criminalidad que se vive en México y de la gobernanza para la seguridad, el ILP constituye una herramienta de gestión que permitiría fortalecer el diseño de estrategias de seguridad pública y un uso eficiente de los recursos, pero ello requiere de cambios que, se están haciendo de manera muy lenta en las entidades federativas.

\section{NOTA SOBRE EL AUTOR:}

Juan Carlos Montero Bagatella es profesor de cátedra e investigador en el Departamento de Ciencias Políticas y Relaciones Internacionales en el Tecnológico de Monterrey, Campus Monterrey. Es licenciado en Ciencias Políticas por la Benemérita Universidad Autónoma de Puebla, en Sociología por la UNAM e hizo sus estudios de posgrado en la Escuela de Gobierno del Tecnológico de Monterrey. Sus principales temas de investigación son políticas de seguridad pública, seguridad nacional y estudios electorales. Correo electrónico: juancarlosmonterob@tec.mx 


\section{REFERENCIAS}

Acosta, Raúl y Chávez, Jorge L. (2012), "El Sistema Plataforma México", en Villa, Luis A. (Coord.), Plataforma México: La expresión tecnológica del Nuevo Modelo de Policía, Ciudad de México: Secretaría de Seguridad Pública Federal.

Burcher, Morgan \& Whelan, Chad (2019), "Intelligence-Led Policing in Practice: Reflections From”, Police Quarterly, Vol. 22, No. 2, pp. 139-160.

Bureau of Justice Assistance (2010), Reducing crime through intelligence-led policing, Washington, D.C.: Department of Justice.

Carter, David L. \& Carter, Jeremy G. (2009), "The intelligence Fusion Process for State, Local and Tribal Law Enforcement", Criminal Justice and Behavior, Vol. 36, No. 12, pp. 1323-1339.

Carter, Jeremy G. (2016), "Institutional Pressures and Isomorphism: The Impact on Intelligence-Led Policing Adoption”, Police Quarterly, Vol. 19, No. 4, pp. 435-460.

Carter, Jeremy G.; Carter, David L. \& McGarrell, Edmund (2017), "Law enforcement fusion centers: Cultivating an Information Sharing Environment while Safeguarding Privacy", Journal of Police and Criminal Psychology, Vol. 32, No. 1, pp. 11-27.

Carter, Jeremy G. \& Phillips, Scott W. (2015), "Intelligence-led policing and forces of organisational change in the USA", Policing and Society, Vol. 25, No. 4, pp. 333-335.

Chalk, Peter \& Rosenau, William (2003), "Intelligence, Policy and Counterterrorism; assesing post-9/11 intiatives", Santa Monica, California: RAND Corporation.

Clarke, Susan E. \& Chenoweth, Erica (2006), “The politics of vulnerability: Constructing local performance regimes for local performance regimes for homeland security", Review of Policy Research, Vol. 17, No. 4, pp. 95-114.

Constitución Política de los Estados Unidos Mexicanos, 2020.

Darroch, Steve \& Mazerolle, Lorraine (2013), "Intelligence-led policing: a comparative analysis of community context influencing innovation uptake", Policing and Society, Vol. 25, No. 1, pp. 1-24.

Esteban, Miguel A. y Navarro, Diego (2003), "Gestión del conocimiento y servicios de inteligencia: la dimensión estratégica de la información”, El Profesional de la Información, Vol. 12, No. 4, pp. 269-281.

Gemke, Pascal; Den Hengst, Mariëlle; Van Rosmalen, Flore \& De Boer, Annete (2019), "Towards a maturity model for intelligence-led policing A case study research on the investigation of drugs crime and on football and safety in the Dutch police", Police Practice and Research. https://doi.org/10.1080/15614263.2019.1689135

Gobernación (2017), "Boletín de prensa sobre el II Seminario Internacional de Políticas Policiales Basadas en la Evidencia: Red Nacional de Analistas para la Seguridad Pública", Secretaría de Gobernación, 21 de noviembre.

Gobierno de México (2019), “Plan Nacional de Desarrollo 2019-2024”, Ciudad de México: Gobierno de México. 
González-López, Manuel (2019), “Understanding policy learning in regional innovation policies: lessons from the Galician case", The European Journal of Social Science Research, Vol. 32, No. 1, pp. 104-118.

Gookins, Amanda (2003), "The role of intelligence in policy making”, SAIS Review, Vol. 28, No. 1, pp. 65-73.

Herrera Lasso, M. (2009), "Inteligencia para la Seguridad Nacional”, en CISEN, Inteligencia y Seguridad Nacional, Ciudad de México: CISEN.

INEGI (2020), Encuesta Nacional de Victimización y Percepción sobre Seguridad Pública 2019, Ciudad de México: INEGI.

James, Adrian (2003), “The Advance of Intelligence-Led Policing Strategies: The Emperor's New Clothes?", The Police Journal, Vol. 76, No. 1, pp. 45-59. https://doi.org/10.1177/0032258X0307600105

Johnson, Charles L. (2010), Police use of intelligence to reduce crime, Washington: LFB Scholarly Publishing.

Johnson, Loch K. (2003), "Prefase to a theory of strategic intelligence", International Journal of Intelligence and Counterintelligence, Vol. 16, No. 4, pp. 638-663. https://doi.org/10.1080/716100470

Ley de Seguridad Nacional, 2019.

Ley General del Sistema Nacional de Seguridad Pública, 2019.

Ley Orgánica de la Administración Pública Federal, 2020.

Ley Orgánica de la Administración Pública (LOAP) de Campeche, 2019.

Ley Orgánica de la Administración Pública (LOAP) de Colima, 2018.

Ley Orgánica de la Administración Pública (LOAP) de Estado de México, 2020.

Ley Orgánica de la Administración Pública (LOAP) de Morelos, 2019.

Ley Orgánica de la Administración Pública (LOAP) de Nuevo León, 2020.

Ley Orgánica de la Administración Pública (LOAP) de Puebla, 2019.

Ley Orgánica de la Administración Pública (LOAP) de Tlaxcala.

Ley Orgánica de la Fiscalía General del estado de Chihuahua, 2010.

Ley Orgánica del Poder Ejecutivo del Estado de Jalisco, 2019.

Liebowitz, Jay (2010), "Strategic Intelligence, Social Networking and Knowledge Retention", Computer, Vol. 43, No. 2, pp. 87-89.

Massarotti, Michael (2012), "Intelligence-led policing: the evaluation of the Denver Police Department's", Journal of Applied Security Research, Vol. 7, No. 2, pp. 268-283. https://doi.org/10.1080/19361610.2012.656259

Moloeznik, Marcos P. y Balcázar, Manuel I. (2013), "Aproximación a la inteligencia policial (la Policía Federal de México, 2006-2012)”, Revista Criminalidad, Vol. 55, No. 1, pp. 131151. 
Navarro, Diego (2004), "El ciclo de inteligencia y sus límites”, Cuadernos Constitucionales de la Cátedra Fabrique Furió Ceriol, No, 48, pp. 51-66.

ONUDC (2011), Criminal Intelligence. Manual for Managers, New York: ONUDC.

OSCE (2017), Guía de la OSCE sobre actividad policial basada en la inteligencia, Viena: Secretaría OSCE.

Peterson, Marilyn (2003), Intelligence-Led Policing: The New Intelligence Architecture, Washington, D.C.: Bureau of Justice Assistance.

Ratcliffe, Jerry H. (2016), Intelligence-Led Policing, New York: Routledge.

Ratcliffe, Jerry H. \& Guidetti, Ray (2008), "State police investigative structure and the adoption of intelligence-led policing", Policing: An International Journal of Police Strategies \& Management, Vol. 31, No. 1, pp. 109-128.

Ratcliffe, Jerry H.; Sorg, Evan T. \& Rose, James W. (2015), "Intelligence-Led Policing in Honduras: Applying Sleipnir and Social Psychology to Understand Gang Proliferation", Journal of Police and Criminal Psychology, Vol. 30, No. 2, pp. 112-123. https://doi.org/10.1007/s11896-014-9143-4

Reglamento Interior de la Secretaría de la Defensa Nacional, 2019.

Reglamento Interior de la Secretaría de Marina, 2015.

Reglamento Interior de la Secretaría de Seguridad Pública (RISSP) de Aguascalientes, 2017.

Reglamento Interior de la Secretaría de Seguridad Pública (RISSP) de Baja California, 2010.

Reglamento Interior de la Secretaría de Seguridad Pública (RISSP) de Campeche, 2018.

Reglamento Interior de la Secretaría de Seguridad Pública (RISSP) de Chiapas, 2018.

Reglamento Interior de la Secretaría de Seguridad Pública (RISSP) de la Ciudad de México, 2020.

Reglamento Interior de la Secretaría de Seguridad Pública (RISSP) de Durango, 2011.

Reglamento Interior de la Secretaría de Seguridad Pública (RISSP) del Estado de México, 2020.

Reglamento Interior de la Secretaría de Seguridad Pública (RISSP) de Morelos, 2017.

Reglamento Interior de la Secretaría de Seguridad Pública (RISSP) de Querétaro, 2020.

Reglamento Interior de la Secretaría de Seguridad Pública (RISSP) de Tamaulipas, 2013, Artículo 12.

Reglamento Interior de la Fiscalía General de Chihuahua, 2018

Reglamento Interno de las Instancias Administrativas del Despacho del Fiscal General de Chihuahua, 2014.

Reppetto, Thomas (2011), Battleground New York City: Countering Spies, Saboteurs, and Terrorists Since 1861, Washington D.C.: Potomac Books. 
SESNSP (2020), Incidencia delictiva del fuero común. Recuperado el 21 de noviembre de 2019, https://drive.google.com/file/d/1FjQuxsKlWSCmsbgMgg5bFBGAz97uAP4x/viewg

Sheremetov, Leonid (2012), "Sistematización y Explotación de la Información", en Villa, Luis A. (Coord.), Plataforma México: La expresión Tecnológica del Nuevo Modelo de Policía, Ciudad de México: Secretaría de Seguridad Pública Federal, pp. 114-140.

Tello, Jorge E. (2012), "Inteligencia estratégica en el contexto mexicano”, en Tello, Jorge E.; Laborde, Adolfo A. y Villarreal, Mario (Eds.), Inteligencia estratégica en el contexto mexicano, Ciudad de México: Plaza y Valdés, pp. 9-12.

Tita, George; Riley, K. Jack; Ridgeway, Greg; Grammich, Clifford; Abrahamse, Allan F. \& Greenwood, Peter W. (Eds.) (2003), Reducing gun violence: Results from an intervention in East Los Angeles, Los Angeles: RAND Corporation.

Torres, Pedro y Santiago, Oscar A. (2015), La inteligencia en el nuevo sistema de justicia penal, Monterrey: INACYPE.

Treverton, Gregory F. (2011), Moving Toward the Future of Policing, RAND Corporation.

Tudela, Patricio (2012), “Achievements and Challenges in Promoting Crime Analysis: Chile's experiences and best practices in South America”, XXII Annual Training Conference of The International Association of Crime Analysts (IACA), Henderson - Nevada, United State. 